\title{
El cambio de paradigma de la industria a través de la reingeniería de procesos
}

\section{The industry paradigm shift through process reengineering}

1 Luis Antonio Chica Castro.

Universidad de Guayaquil, Guayaquil, Ecuador luis.chicac@ug.edu.ec

2 Hugo Ernesto Solís Ferrer.

Universidad de Guayaquil, Guayaquil, Ecuador hugo.solisf@ug.edu.eg
iD https://orcid.org/0000-0002-1560-5147

https://orcid.org/0000-0002-7197-8319

Artículo de Investigación Científica y Tecnológica

Enviado: 12/12/2021

Revisado: 28/12/2021

Aceptado: 28/01/2025

Publicado:05/02/2022

DOI https://doi.org/10.33262/ap.v4i1.1.160

a través de la reingeniería de procesos. AlfaPublicaciones, 4(1.1), 293-310.

Cítese: $\quad$ https://doi.org/10.33262/ap.v4i1.1.160

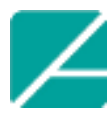

Ciencia

Digital

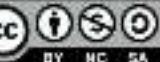

ALFA PUBLICACIONES, es una Revista Multidisciplinar, Trimestral, que se publicará en soporte electrónico tiene como misión contribuir a la formación de profesionales competentes con visión humanística y crítica que sean capaces de exponer sus resultados investigativos y científicos en la misma medida que se promueva mediante su intervención cambios positivos en la sociedad. https://alfapublicaciones.com

La revista es editada por la Editorial Ciencia Digital (Editorial de prestigio registrada en la Cámara Ecuatoriana de Libro con No de Afiliación 663) www.celibro.org.ec

Esta revista está protegida bajo una licencia Creative Commons Attribution Non Commercial No Derivatives

4.0 International. Copia de la licencia: http://creativecommons.org/licenses/by-nc-nd/4.0/ 
Palabras

claves:

reingeniería, paradigma, cambio, procesos.

\section{Keywords:} reengineering, paradigm, change, processes

\section{Resumen}

La historia de la evolución humana, siempre ha estado impactada de cambios de paradigmas (rupturas de modelos, creencias o costumbres), en la búsqueda de mejoras y comodidades para el ser humano. Estos procesos de cambios de paradigma, también sucede con las industrias que inicialmente pudieron surgir como pequeñas empresas familiares o empresas sin mayores aspiraciones, pero que con el correr del tiempo les ha correspondido adaptarse a fin de sobrevivir a la globalización y a otras innovaciones tecnológicas, siendo la reingeniería de proceso una alternativa capaz de repesar los procedimientos que se realizan en las industrias, revisándolos desde cero y con ello rompiendo paradigmas para su aplicación. Sin embargo, es importante conocer cuándo, cómo y porqué implementarla a fin de lograr el éxito de la misma., siendo esto la finalidad de la presente investigación, usando para ello, una metodología basada en el diseño bibliográfico de tipo documental. Obteniendo al final, algunas conclusiones al respecto que dan luz sobre como suceden los cambios de paradigma en la industria al hacer uso de la reingeniería de procesos.

\section{Abstract}

Human evolution history has always been impacted by paradigm shifts (ruptures of models, beliefs or customs), in the search for improvements and comforts for the human being. These processes of paradigm shift also happen with industries that could initially emerge as small family businesses or companies without major aspirations, but which over time have had to adapt in order to survive globalization and other technological innovations, being process reengineering an alternative capable of reviewing the procedures carried out in industries, reviewing them from scratch and thereby breaking paradigms for their application. However, it is important to know when, how and why to implement it in order to achieve its success, this being the purpose of this research, using a methodology based on the bibliographic design of a documentary type. Obtaining at the end, some conclusions in this regard that shed light on how paradigm shifts occur in industry when making use of process reengineering. 


\section{Introducción}

Las empresas a lo largo del tiempo, han tenido que romper con los paradigmas tradicionales para poder sobrevivir a la globalización, las nuevas tecnologías, las demandas cada vez más exigentes de la sociedad y de las competencias de empresas similares.

Esta ruptura del paradigma tradicional, involucra una serie de cambios, indica Procel (2016), que la mayoría de las empresas requieren cambios importantes en el comportamiento y la filosofía de la dirección, para sobrevivir en el fluido y dinámico ambiente empresarial de la actualidad.

Asimismo, Díaz (2018) afirma que, el mundo empresarial está rodeado de cambios tan vertiginosos en todos los puntos de vista, que las organizaciones se ven impulsadas quieran o no, a tomar iniciativas de adaptación organizacional para mantener sus labores.

De ahí, que el cambio es una necesidad inaplazable en el sistema empresarial para adaptarse a los distintos escenarios económicos, tecnológicos, comerciales y laborales en los que se mueven las empresas en la contemporaneidad para garantizar no sólo su supervivencia, sino desarrollarse y conquistar nuevos mercados (Moreno \& Parra, 2017).

Por ello, es importante, tener presente que las organizaciones deben orientar sus esfuerzos hacia la satisfacción de las necesidades de sus clientes y, a través de ello, lograr una mayor rentabilidad, operando en un entorno que se transforma de manera permanente lo cual las obliga a responder activamente a los cambios sin afectar la calidad de sus productos y/o servicios (Hernández, 2019).

Uno de los procedimientos que hacen posible estos cambios desde sus bases hasta la gerencia de las empresas es la Reingeniería, la cual según Procel (2016), como toda actividad novedosa ha recibido diversidad de nombres, entre ellos: modernización, transformación y reestructuración, pero independientemente del nombre, la meta es siempre la misma: aumentar la capacidad para competir en el mercado mediante la optimización de los costos.

La reingeniería de procesos puede suponer una gran ventaja competitiva para las empresas, dado que, si se realiza de forma adecuada, rediseñando los procesos para obtener mejoras, aumentando así el rendimiento e incluso reduciendo costes (Pérez et al., 2017).

Sin embargo, a pesar de los beneficios que ofrece, reducción de costo, satisfacción al cliente, mantenerse en el mercado, entre otras, algunas empresas temen incorporar a sus procesos la reingeniería, ya que esta herramienta se basa en la restructuración desde cero de sus procesos, es decir no es mejorar el proceso, es transformarlo. Indica Morales 
(2019), "la reingeniería no es cuestión de hacer mejoras marginales o incrementales sino de dar saltos gigantescos en rendimiento".

$\mathrm{Y}$ es en esa transformación donde entran en juego las creencias, las costumbres, el considerar que es mejor que todo siga igual, solo por el temor al cambio radical. Ya que como menciona Díaz (2018), es estar ante una situación en donde se dejan determinadas estructuras, procedimientos, comportamientos, para adquirir otras.

Además, agrega el autor anterior, todo cambio organizacional, rompe rutinas y paradigmas de actuación por ello, la reingeniería representa una posibilidad de cambio, pues plantea el abandono de los viejos procesos y la búsqueda de nuevos paradigmas que agreguen valor, rompiendo la antigua estructura y cultura de trabajo.

A fin de lograr el éxito de la reingeniería y evitar dichos temores, es importante el apoyo total de la compañía, desde la gerencia hasta su línea de empleados. Para Procel (2016), la alta dirección debe hacerse consiente de las diversas fases de la Reingeniería y el impacto potencial que podría tener esta sobre la estructura, cultura y recursos de la empresa.

En consideración, si la industria quiere sobrevivir a los evidentes cambios tecnológicos y a la demanda cada más exigente de la sociedad, debe romper paradigmas y lograr que todo su personal se adapte a los cambios que se realizan con la implementación de herramientas como la reingeniería de procesos.

\section{Metodología}

Para la elaboración del presente estudio, se utilizó una metodología de tipo documental, con base en el diseño bibliográfico. Según Rojas (2013), para la investigación documental se recurre a las fuentes históricas, monografías, información estadística y a todos aquellos documentos que existen sobre el tema para efectuar el análisis del problema.

Además, la revisión bibliográfica permite garantizar la obtención de la información más relevante en el campo de estudio, de un universo de documentos que puede ser muy extenso (Gómez et al., 2014).

Al considerar diferentes documentos tales como libros, revistas, tesis, entre otros, prevaleció como criterios para la selección de textos, la pertinencia de los mismos con el objeto de estudio, tomando solo aquellos que cumplieran con veracidad de la información en fuentes confiables, permitiendo de esta manera sustentar teóricamente el estudio.

Todo lo anterior permitió conocer y analizar las diferentes posiciones de los autores consultados y de esta manera plantear la importancia que tienen la reingeniería para las empresas e industrias. 


\section{Resultados y Discusión}

Durante el recorrido bibliográfico para el desarrollo de esta investigación, se pudo evidenciar que los diferentes autores coinciden desde sus percepciones en la importancia de la reingeniería de procesos para las industrias, considerando este hecho se desglosan los aspectos que se presentan a continuación.

\section{La Reingeniería de Procesos}

La conceptualización de la reingeniería de procesos pasa por diferentes connotaciones, pero siempre se diluye en que es la transformación, o restructuración radical de los procesos que se ejecutan en la empresa, y es precisamente esta connotación que la hace diferente a otras herramientas.

Así lo sostiene Bustos (2010), mencionado por Moreno \& Parra (2017), la reingeniería de procesos proporciona un enfoque global al rediseño y reconstrucción de los procesos de una organización y no debe ser confundida con otros enfoques como Gestión por Procesos, Downsizing, Reingeniería de Software, Calidad Total o Mejora Continua.

Para Hernández (2019), la reingeniería es el rediseño de procesos en un negocio o un cambio drástico de un proceso; es comenzar de nuevo desde cero, es un cambio de todo o nada. Por eso, indica el mismo autor, es una herramienta que la utilizan para volver a crear nuevos enfoques de las estructuras de los procesos basándose en nuevas teorías y técnicas. Busca tener avances decisivos no solamente completarlos y cambiarlos. Es transformar lo antiguo en algo nuevo.

Siendo lo anterior la base fundamental de esta herramienta, según la definición de sus creadores, mencionado por Pilco (2020) y por Hernández (2019), la reingeniería es la revisión fundamental y el rediseño radical de procesos de negocios para alcanzar mejoras espectaculares en medidas críticas y contemporáneas de rendimiento, tales como costos, calidad, servicio y rapidez.

Es decir, la reingeniería de procesos es una herramienta útil que sirve para analizar los procesos de una empresa y así poder formular nuevos procesos (Pérez et al., 2017).

En ese orden de ideas, para Díaz (2018), es una herramienta de cambio que tiene como objetivos específicos aumentar la productividad, optimizar el valor de la organización para todos sus integrantes.

En opinión de Morales (2019), la reingeniería se trata del rediseño total de los procesos para brindar mejoras en la organización en sus costos, calidad, servicio y rapidez, todo dirigido a la satisfacción al cliente. Es modificar un proceso que falla y está afectando la producción, pero la reingeniería no brinda soluciones parciales, por el contrario, revoluciona la forma de administrar los procesos, puesto que no los mejora de manera 
superflua, sino que los rediseña desde el principio, sin fijarse en prejuicios pasados (Gordon, 2017).

A la luz de todas las aserciones anteriores, se puede decir que la reingeniería, no es una modificación de un proceso dentro de la industria que ha presentado alguna falla o que no cubre la línea de producción en el tiempo estipulado, tampoco es una moda que se debe tomar, es un cambio total de ese proceso, sin mirar atrás, es analizarlo para cambiarlo totalmente.

A veces sucede que se culpa de esas fallas de la línea de la producción, a los empleados que trabajan en ese proceso o al encargado del equipo aludiendo fallas de la maquina por mantenimiento, sin detenerse a buscar o analizar la raíz del problema que puede ser la forma como se ejecuta dicho proceso. Afirma Procel (2016), se culpa a los empleados, a los encargados o la maquinaria cuando las cosas no marchan bien, cuando en realidad la culpa no es de ellos sino de la forma en qué se trabaja, y agrega el autor, también es importante hacer notar que no es porque el proceso sea malo, sino que es malo en la actualidad debido a que el proceso fue diseñado para otras condiciones de mercado que se daban en el pasado.

Por lo tanto, es importante resaltar la importancia que tiene la reingeniería para las industrias, para la obtención de mayores beneficios de sus procesos, pero no se puede tomar a la ligera ya que involucra la transformación total de los mismo, por eso es importante realizar una investigación y análisis de la situación antes de tomar la decisión de implantarla.

Con base a lo anterior, reingeniería tampoco es comprar computadoras para estar a la moda o simplemente cambiar el color al local y los uniformes a los empleados, es rediseñar radicalmente lo que se ha venido haciendo para obtener resultados extraordinarios (Morales, 2019).

\section{Pasos a seguir para la implementación de la reingeniería de procesos}

En este punto se pudo evidenciar que no existe un procedimiento estandarizado para realizar reingeniería, que los pasos a seguir deben estar pensados en el proceso que se desea transforma, es decir, como indica Moreno \& Parra (2017), debe ser particularizado y contextualizado en función de lo que se desea rediseñar.

Asimismo, indica Enríquez \& Cadena (2019) que, en la actualidad, aunque exista la voluntad de cambio, la metodología que se requiere para hacerlo no es muy conocida, las empresas deben aprender a cambiar por sí misma de una manera más eficaz que en el pasado. 
Moreno \& Parra (2017), también se unen a las afirmaciones anteriores al indicar que la reingeniería se presenta en una secuencia de pasos generales, sin describir las posibles técnicas a aplicar o procedimientos a seguir, debido a las particularidades del contexto empresarial donde se aplique la reingeniería, y a intereses comerciales y legales. De igual manera, Gordon (2017) expresa al decir que, son diversos los métodos para gestionar la reingeniería de procesos, sin embargo, no existe una fórmula mágica general que funcione de manera general en todas las organizaciones.

Pero pese a lo anterior, algunos de los autores se atreven a proponer etapas o pasos generales para la aplicación de la reingeniería de procesos, como los que se presentan a continuación:

Procel (2016), basa estas etapas en lo mencionado por los precursores de la reingeniería Hammer y Champy, asegurando que existen cinco pasos muy importantes a la hora de realizar la reingeniería:

- Diseño del mapa de procesos. Permite evidenciar las tareas, los roles, la relación entre áreas de la organización, tiempos de ejecución y los responsables.

- Elaborar un inventario de los procesos. Es la identificación de los procesos y chequear y definición de cada proceso. Es importante mencionar que los procesos pueden estar definidos como estratégicos, cuya responsabilidad es de la gerencia; procesos de apoyo son necesarios para el control, mejora y soporte de la cadena de negocio y por último los agregadores de valor, los mismos son necesarios para la realización del producto.

- Caracterización de los procesos. Permitirá identificar los elementos esenciales para llevar a cabo el proceso y la definición de las principales características, facilitando su entendimiento, gestión y el control de sus interrelaciones.

- Modelamiento de los procesos. Se refiere al conjunto de actividades, personas, roles y reglas que permiten articular los procesos y servicios de la empresa hacia el exterior (clientes, proveedores) y hacia el interior (otros departamentos). Además, permite dibujar y documentar los procesos de la organización, acompañado de actividades de toma de información y análisis que facilitan la detección de errores en los procesos y su optimización progresiva.

- Desarrollo del flujograma de cada uno de los procesos los mismos que serán plasmados en el Manual de Procedimientos (Procel, 2016).

Pérez et al. (2017), considera que la reingeniería debería ser un método para aprovechar las fortalezas internas de le empresa, y eliminar las debilidades de la misma, aprovechado oportunidades externas, esa metodología se basaría en lo siguiente: 
- Definir el proyecto: es decir, que alcance tiene el proyecto y cuáles son los objetivos o metas que plantea.

- Análisis de la situación: para ello se debe realizar un diagnóstico de la situación actual de la organización, evaluando:

a. La organización: Historia, tecnología, productos y servicios ofrecidos, recursos utilizados, estrategias y políticas, prospectiva, estructura, entre otros.

b. Entorno: Conocer la demanda de los productos ofrecidos, así como las características de los clientes, competidores, proveedores.

c. Flujo de procesos: Información documentada de procesos tanto administrativos como técnicos.

d. Paradigmas empresariales: Estudiar supuestos conscientes e inconscientes de la empresa y cuestionar los supuestos que no son válidos.

- Diagnóstico: donde se refleje cuáles son las necesidades más urgentes de la institución, limitaciones y debilidades para poder llevar una gestión eficiente.

- Diseño de la nueva organización: se recoge información de las etapas anteriores, y se crea una organización que cubra necesidades y limitaciones de la organización actual. Debe reflejar:

a. Flujos de procesos: Los procesos que necesitan rediseño.

b. Flujos de información: Documentos, archivos o sus nuevos flujos para que la información fluya con un adecuado sistema de información gerencial.

c. Organización: Diseño de características generales de la nueva estructura organizacional, tales como cargos, funciones, mercadeo de servicios, gestión financiera, cargas de trabajo y cultura organizacional.

d. Estrategias y políticas: Nuevo sistema de gestión que va a seguir la organización.

e. Paradigmas empresariales: Nuevas creencias y formas de hacer las cosas.

f. Plataforma tecnológica: Determinar características y configuraciones necesarias para el software y el hardware.

g. Productos o servicios: Proporcionar el producto o servicio con las características fundamentales para satisfacer las necesidades del cliente

- Implementación del nuevo diseño de la organización. Puesta en marcha del nuevo diseño o proyecto de organización.

- Validación del buen funcionamiento de las mejoras implantadas, a través de una serie de indicadores para medir el correcto desarrollo de los procesos rediseñados.

- En caso de existir deficiencias o falta de alguna mejora, se realizaría un rediseño de la organización hasta alcanzar los objetivos establecido (Pérez et al., 2017). 
Pérez et al. (2017), también mencionan los pasos de ejecutados por Escobar y González, en un caso práctico de implementación:

- Inducción a la reingeniería: periodo en el cual la alta gerencia debe persuadir a los integrantes de la organización de la necesidad de afrontar cambios importantes en la misma.

- Identificación y análisis de oportunidades de rediseño: Consiste en identificar que procesos necesitan ser rediseñados. Existen criterios para priorizar en unos procesos sobre otros a la hora de rediseñarlos: procesos que presentan problemas, importancia o impacto sobre clientes externos, viabilidad de que se puedan realizar rediseño con éxito.

- Rediseño de los procesos: Es la parte más creativa, en la cual hay que innovar o rediseñar los procesos para que estos sustituyan a los procesos anteriores.

- Implantación de los procesos rediseñados: Consiste en sustituir los procesos anteriores por los actuales, ya sean completamente nuevos o actualizaciones de los anteriores.

Para Moreno \& Parra (2017), la aplicación de la reingeniería de procesos a cualquier organización que se dispusiera a rediseñar sus procesos estratégicos, debe dar especial atención a los siguientes aspectos:

- Utilización óptima de los recursos materiales, financieros y de capital humano.

- Reingeniería orientada al cliente y a la satisfacción de sus necesidades y expectativas.

- Maximizar los procesos que aportan valor al cliente y a la organización.

- Reducción de costos y gastos permitiendo potenciar los resultados económicos de la organización.

- Minimizar los riesgos asociados a deficiencias en los procesos y los sistemas de dirección y gestión empresarial.

- Utilización de las tecnologías de la información en función de los procesos rediseñados.

- Los impactos cuantitativos y cualitativos son base para la evaluación de los resultados de su aplicación.

Estos autores además implementaron un caso práctico aplicando el flujograma de la figura 1, el cual coincide con algunos de los pasos acá planteados. 


\section{Figura 1}

Diagrama general para la implementación de la reingeniería

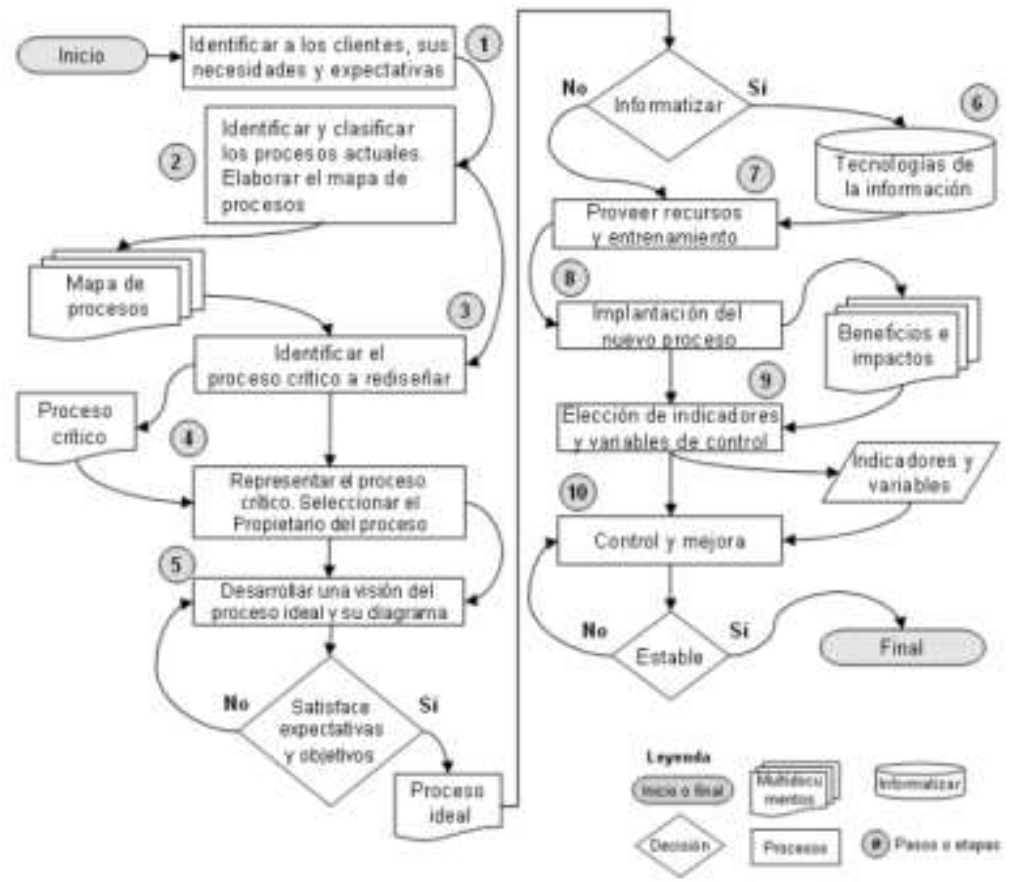

Fuente: Moreno \& Parra (2017)

Como se puede observar existe mucha coincidencia en los documentos de los diferentes autores analizados, en lo que respecta a los pasos o pautas a seguir para la aplicación de la reingeniería, indicando que siempre no son procesos tácitos, sino más bien flexibles a cada situación de la organización donde sea necesaria la reingeniería.

Por ello, la reingeniería de procesos independientemente de la metodología utilizada persigue el mismo objetivo, alcanzar la meta deseada con respecto a la optimización de los procesos, consiguiendo siempre una implicación total del personal en los cambios propuestos para la mejora de la organización (Pérez at el., 2017).

Sin embargo, se hace necesario tomar en cuenta los siguientes aspectos cuando se haga reingeniería de procesos, según Enríquez \& Cadena (2019):

- Cualquier cambio importante es para mejorar en la productividad, y reducir costos de producción; realizar mejoras en la calidad del servicio; garantizar el crecimiento, la innovación, la diversificación de la empresa, además agregar valor para los clientes y accionistas y otros interesados en la empresa.

- El itinerario a seguir en la organización es el enfoque hacia los procesos, caracterizada por el reconocimiento de la organización del trabajo dado por grandes funciones y procesos, debe superar los límites de estos, empezando desde el punto cero en lugar de tratar de cambiar lo ya existente, estos procesos y programas de 
cambio deben llevarse a cabo de arriba abajo impulsados por una visión con amplia participación de todos los implicados en las fases de diseño y de ejecución (Enríquez \& Cadena, 2019)

\section{Características de la reingeniería}

Procel (2019) menciona, que una de las características principales de la reingeniería de procesos es la unificación de varias tareas y como consecuencia se logra una reducción de plazos, al eliminarse supervisiones, a la vez que se mejora la calidad, al evitarse errores. Por otra parte, la reingeniería produce cambios y estos se reflejan según el mismo autor en:

Cambio de la función del trabajador: los trabajadores toman las decisiones y asumen las responsabilidades relacionadas con su trabajo, es decir cada empleado se convierte a su vez en su propio jefe. Para que esto se pueda llevar a cabo son necesarios el esfuerzo, el apoyo, la disciplina, la confianza, la flexibilidad y la capacidad de adaptación.

Cambios del orden: una vez introducida la reingeniería, las actividades se comienzan a realizar en el orden en que se beneficie a los procesos, olvidándose del orden tradicional.

Cambios en los controles y comprobaciones: a través de la reducción de las comprobaciones y controles se trata de establecer un plan de evaluación y control que contemple solamente los controles que tienen sentido económico.

A la luz de estas características, es evidente que la reingeniería es sinónimo de cambio, de transformación y de allí es importante que la reingeniería de procesos tiene un importante impacto en las industrias, tanto en sus estructuras como el personal involucrado en dichos procesos y ese impacto genera una ruptura de los paradigmas existentes.

\section{Cambio de Paradigma a través de la Reingeniería}

Desde el punto de vista holístico, la concepción de paradigma viene dada por el conjunto de posiciones que puede tener en un determinado momento el ser humano, pasando desde su sistema de creencias e ideas, hasta su forma de pensar y actuar.

Para Saldaña (2019), un paradigma es el supuesto colectivo de las creencias y actitudes en una época que, proporciona al ser humano una manera de ver el mundo, de situarse y actuar en él. Además, indica el autor, que un paradigma no es eterno, en tanto el sistema de creencias y actitudes que conforman la cosmovisión del mundo de un grupo humano en una época, cambia por hechos de la misma realidad humana que van aconteciendo y que exigen nuevas miradas. 
Es decir, los paradigmas no se mantienen en el tiempo, debido a los cambios que van surgiendo en pro de mejoras para la calidad y comodidad del ser humano, y es lo que ha sucedido con el pasar del tiempo, donde los seres humanos han cambiado sus ideas, creencias y percepciones por nuevos modelos, por nuevos paradigmas.

Por otra parte, actualmente el término paradigma es usado en diferentes áreas y su definición exacta por ende depende de área desde donde se esté aplicando. Sin embargo, en general hablar de un paradigma es hablar de algo ejemplar, o sea, de algo característico de una visión o perspectiva, o bien algo que resume un sistema de pensamiento o de hacer las cosas (Equipo editorial Etecé, 2021)

En base a lo mencionado anteriormente, en referencia al cambio de posturas del ser humano o la transformación del modelo, es lo que se le conoce como cambio de paradigma. Para Kuhn mencionado en el artículo del Equipo editorial Etecé (2021), los cambios de paradigma se producen cuando un modelo fracasa en su labor de interpretar determinados aspectos de la realidad.

Es decir, un cambio de paradigma es un cambio de perspectivas, de métodos o de tendencias a gran escala, aplicable a cualquier otra área del saber o de la experiencia humana, también puede suceder que la realidad cambia de modo tal que obliga a producir una nueva cosmovisión, dado que el paradigma no es sólo una teoría vigente, sino un sistema entero de creencias y presuposiciones (Equipo editorial Etecé, 2021).

Con base a lo planteado, la reingeniería de procesos no es en sí misma un paradigma. Ella, sin embargo, requiere de un nuevo paradigma para ser eficaz (Enríquez \& Cadena, 2019). A la luz de esas afirmaciones, para el éxito de la reingeniería, es necesario un cambio de paradigma, un cambio de los modelos de procesos que siguen las industrias y con ello un cambio en el sistema de creencias y costumbres que tiene de esos procesos los empleados y hasta la alta gerencia de la industria.

Es importante por ello tener en cuenta que, todo cambio trae consigo, temor y por ende resistencia, esto puede suceder al implementar la reingeniería de procesos, cuando los trabajadores tienen el temor a que las cosas no salgan con los resultados esperados o a que sencillamente no quieren cambiar sus métodos antiguos por costumbres marcadas por muchos años laborables. Indica Morales (2019), normalmente la mayor barrera para el cambio lo constituye la resistencia al cambio de los empleados de la organización, ya que por años o tradicionalmente han trabajado de una forma, que para ellos ha resultado buena, mas no necesariamente para la empresa.

Siendo así, la resistencia al cambio es una de las principales causas de fracaso de la reingeniería de procesos en la industria, así que se de contar con el compromiso de la alta 
gerencia, además de un buen liderazgo, una amplia comunicación y participación de los empleados.

En este sentido Morales (2019) menciona que:

Es por esto que el compromiso de los líderes debe ser al 100\% con la reingeniería y dejar atrás las costumbres. Sin embargo, se ha comprobado que la gente no es reacia al cambio en sí mismo, sino a la forma en que se efectúa el cambio, ya que en muchas ocasiones existe una verdadera falta de liderazgo y una mala delegación de responsabilidades del cambio.

Es decir, debe dentro del procesos de reingeniería debe existir una planificación de cambio, por ende, los ingenieros, jefes y gerentes deben de alguna manera romper el paradigma del o de los procesos a cambiar. Afirma Díaz (2018), el cambio debe redefinir creencias, actitudes, valores, estrategias y prácticas.

Pérez et al. (2017), presentan dentro de los pasos de la implantación de reingeniería etapas similares a las ya mencionadas por otros autores, pero las identifica como cambios y hace especial énfasis como primer paso a la preparación del cambio, que consiste en mentalizar al personal que compone la organización de la necesidad de cambio para la consecución de los objetivos fijados por la empresa.

Las otras etapas las identifica los autores mencionados, basadas en el cambio como: Planeación del cambio: basada en el estudio de las diferentes áreas de la empresa con el objetivo de identificar aquellos procesos y áreas de la empresa en las cuales existe una necesidad urgente de cambio; Diseño del cambio: se realiza el rediseño de los procesos productivos que necesitan mejora urgente, así como implantación de las modificaciones; Evaluación del cambio: es la validación del rediseño o mejora de los procesos durante un periodo de tiempo en el cual se pueden corregir errores en dichos rediseños o mejoras.

Dando por sentado que los cambios dentro de la industria son necesarios, que siempre han ocurrido, pero ahora, debido a la globalización y a los cambios tecnológicos se hace necesario adaptarse al cambio de manera más rápida, indica Díaz (2018), las organizaciones exitosas deben estar abiertas al cambio, ser competitivas y vivir orientadas totalmente hacia los clientes.

Asimismo, Hammer y Champy precursores de la reingeniería mencionados por Enríquez y Cadena (2019), señalan que algunos de los errores más comunes que llevan a las organizaciones a fracasar es el tratar de corregir un proceso en vez de cambiarlo, esto sucede cuando:

- No se centra la atención en los procesos.

- No se deja de pensar en lo que no es reingeniería de procesos. 
- No se toma en cuenta los valores y las creencias de los empleados.

- Conformarse con resultados de poca importancia.

- Abandonar el esfuerzo antes de tiempo.

- Limitar de antemano la definición del problema y el alcance del esfuerzo de reingeniería.

En ese sentido, como se indicó en anteriores puntos, la reingeniería de procesos no es una moda y su aplicación es drástica, por lo tanto, es importante no solo saber cómo se puede usar o cuales son los pasos a seguir antes, durante y posterior a la implementación, es menester saber cuándo es conveniente usarla a fin de evitar fracasos posteriores, según Pilco (2020), la reingeniería de procesos es una herramienta gerencial que se han aplicado y ha habido casos de éxitos y fracasos al aplicar la reingeniería de procesos.

Considera Rodríguez (2019), la reingeniería de procesos se debe aplicar cuando:

- El rendimiento de la organización está por detrás de la competencia.

- La organización está en crisis; como una caída en el mercado.

- Las condiciones del mercado cambian; como por ejemplo nueva tecnología.

- Se quiere obtener una posición de líder del mercado.

- Hay que responder a una competencia agresiva.

- La empresa es líder y sabe que debe seguir mejorando para mantener el liderazgo.

\section{Conclusiones}

A través de la presente investigación, una vez revisada la bibliográfica y analizados los diferentes autores, se pudo evidenciar que cuando una industria o empresa se está alejando de sus objetivos y metas deben aplicar medidas contundentes a fin de activarse rápidamente al medio y sobrevivir, esto es posible usando la reingeniería de procesos. Por esta razón, se disciernen las siguientes aproximaciones epistemológicas:

- La reingeniería de procesos, es una herramienta que permite hacer cambios drásticos y rápidos en las industrias, pero aún existe temor a implementarla, es decir el cambio no solo es de los procesos estructurales de la industria que puedan presentar problemas, sino que también incluye un cambio de mentalidad de todo el cuerpo gerencial y ejecutor de esos procesos, es decir, un cambio total de paradigma.

Para Procel (2016), la reingeniería constituye una recreación y reconfiguración de las actividades y procesos de la empresa, lo cual implica volver a crear y configurar de manera radical él o los sistemas de la compañía a los efectos de lograr incrementos 
significativos y en un período corto de tiempo, en rentabilidad, productividad, tiempo de respuesta y calidad, lo cual implica la obtención de ventajas competitiva.

Por otra para Díaz (2018) afirma que, la reingeniería es una estrategia gerencial destinada a romper los paradigmas existentes, para innovar y crear nuevas formas de realizar las actividades, logrando ventajas competitivas.

- A fin de evitar el miedo al cambio es conveniente mantener informado a todas las instancias involucradas en la implantación de la reingeniería. Es decir, antes de la implementación de la propuesta, se recomienda compartir la información de los cambios y aspectos de ésta a los empleados de la empresa (Garzón \& Martínez, 2018)

- También es importante analizar el impacto no solo del personal, sino el impacto en los procesos industriales, en la información, en lo tecnológico, en fin, en todas las áreas afectadas para prever posibles acciones a tomar en caso de ser necesario antes de implementarla. Afirma Enríquez \& Cadena (2019), un enfoque de reingeniería debe proveer la habilidad para analizar el impacto que los cambios de cualquier proceso tendrán en todas las unidades organizacionales.

- Una vez implementada la reingeniería, se deben realizar planes que permitan mantenerla en el tiempo. Recomiendan Garzón \& Martínez (2018) que la reingeniería implementada debe contemplar la creación de un plan de mantenimiento, un plan maestro de producción y una redistribución en planta.

- También debe existir un plan de capacitación o actualización de los procesos industriales para el personal, que permita mejorar el nivel de acción y mejoramiento en la producción.

- La reingeniería mejora los procesos a su máxima capacidad, al igual que las funciones del personal, permitiendo la satisfacción de los clientes al tener productos o servicios de calidad. Manifiesta Moreno \& Parra (2017), en el proceso rediseñado se maximizan las actividades y funciones que agregan valor para el cliente y minimizan o eliminan aquellas que no lo hacen, cubriendo necesidades y expectativas de los clientes internos y externos.

- La reingeniería de procesos es una herramienta muy útil y adaptable a cada organización en lo que respecta a los pasos a seguir, es decir, es flexible y dependerá de la persona o grupo de ingeniería que va a implantarla los caminos a seguir para su éxito total. La reingeniería permite conocer la necesidad de cambio en algunos procesos productivos de una empresa, estudiarlos y plantear diferentes metodologías que permitirán mejorar rendimiento de los procesos con la implantación de mejoras y con su posterior validación (Pérez et al., 2017). 


\section{Referencias Bibliográficas}

Díaz, M. (2018). Reingeniería: Una opción de cambio para la actual Escuela de Administración y Contaduría Pública en La Universidad de Carabobo. Estudios Gerenciales y de las Organizaciones, 2 (3), 123-141.

Enríquez, A., \& Cadena, E. (2019). Modelo gerencial basado en la reingeniería. Dilemas contemporáneos: Educación, Política y Valores 7(50) (50).

Equipo editorial Etecé. (17 de febrero de 2021). Paradigma.

Garzón, K., \& Martínez, B. (2018). Propuesta de reingeniería de procesos para la Empresa Balones Milago. Universidad Agustiniana, Facultad de Ingeniería. Bogotá, Colombia: Trabajo especial de grado de la Universidad Agustiniana para optar al título de Ingeniería Industrial.

Gómez, E., Fernando, D., Aponte, G., \& Betancourt, L. (2014). Metodología para la revisión bibliográfica y la gestión de información de temas científicos, a través de su estructuración y sistematización. Dyna, 81 (184), 158-163.

Gordon, J. (2017). Propuesta de Reingeniería de Procesos en el área Logística de la Empresa de Pinturas y Estucos Tex \& Color (Bachelor's tesis, Universidad Autónoma de Occidente)., Facultad de Ingeniería. Santiago de Cali: Trabajo especial de grado de la Universidad Autónoma de Occidente.

Hernández, S. (2019). Reingeniería de procesos en Empresa Techsoft área de desarrollo Banco Guayaquil. Universidad Católica de Santiago de Guayaquil, Facultad de Ingeniería. Guayaquil, Ecuador: Trabajo especial de grado de la Universidad Católica de Santiago de Guayaquil para optar al título de Ingeniería en Sistemas Computacionales.

Morales, A. (18 de febrero de 2019). Reingeniería como movimiento hacia un nuevo paradigma organizacional.

Moreno, R., \& Parra, S. (2017). Metodología para la reingeniería de procesos Validación en la empresa Cereales" Santiago". Ingeniería Industrial 38(2), 38 (2), 130-142.

Pérez, G., Gisbert, V., \& Pérez, E. (2017). Reingeniería de procesos. 3C Empresa: investigación y pensamiento crítico (1), 81-91.

Pilco, G. (2020). Reingeniería de procesos y la gestión administrativa en TECNOMIN DATA, 2019. Universidad Cesar Vallejo, Escuela de Posgrado. Lima, Perú: Trabajo especial de grado de la Universidad César Vallejo. 
Procel, P. (2016). Reingeniería de procesos para mejorar la eficiencia empresarial, en la Empresa PROVISUR del cantón Chambo, provincia de Chimborazo, período 2016. Escuela Superior Politécnica de Chimborazo. Riobamba. Ecuador: Trabajo especial de grado de la Escuela Superior Politécnica de Chimborazo para optar al titulo de Ingeniería en Contabilidad y Auditoría C.P.A.

Rodríguez, J. (11 de diciembre de 2019). Re ingeniería ¿Por qué es importante aplicarla? Consulting Group: https://spcgroup.com.mx/re-ingenieria-por-que-esimportante-aplicarla/

Rojas, R. (2013). Guía para la realización de investigaciones sociales. España: Plaza y Valdez.

Saldaña, J. (2019). ¿Qué es y desde dónde se hace la teología? Preguntas antiguas y respuestas siempre nuevas. Pontificia Universidad Javeriana, Facultad de Teología. Bogotá. Colombia: Trabajo especial de grado de la Pontificia Universidad Javeriana

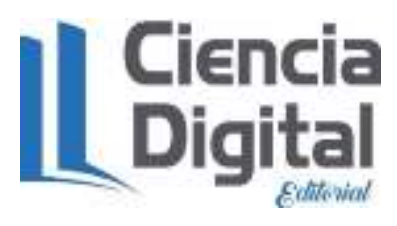


El artículo que se publica es de exclusiva responsabilidad de los autores y no necesariamente reflejan el pensamiento de la Revista Alfa Publicaciones.

\section{Ciencia}

El artículo queda en propiedad de la revista y, por tanto, su publicación parcial y/o total en otro medio tiene que ser autorizado por el director de la Revista Alfa Publicaciones.
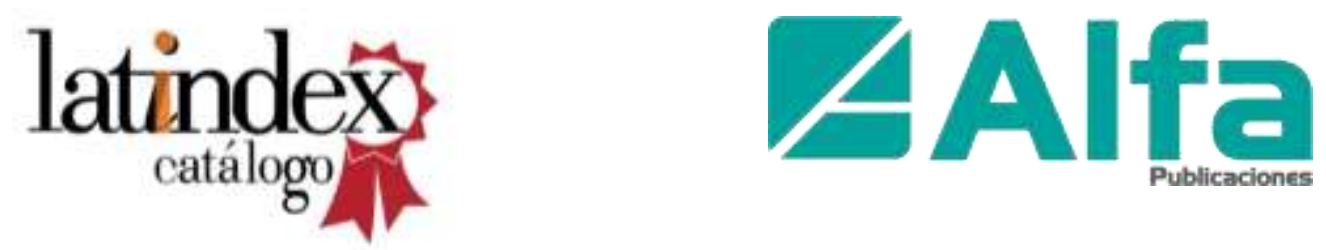

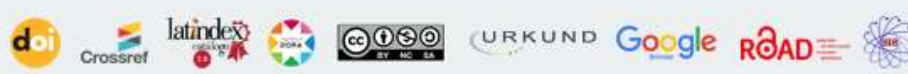
DLatinREV

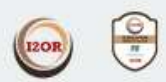

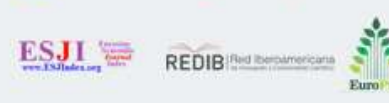

$\underbrace{}_{\text {wizdom.ai }} \mathrm{O}_{\text {OpenAIRE }}^{+}$

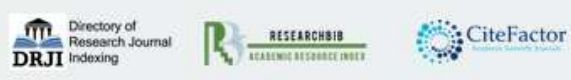

